Acta Crystallographica Section E

Structure Reports

Online

ISSN 1600-5368

\section{2-Naphthyl quinoxalin-2-yl ether}

\section{Noor Doha Hassan, Hairul Anuar Tajuddin, Zanariah Abdullah and Seik Weng Ng*}

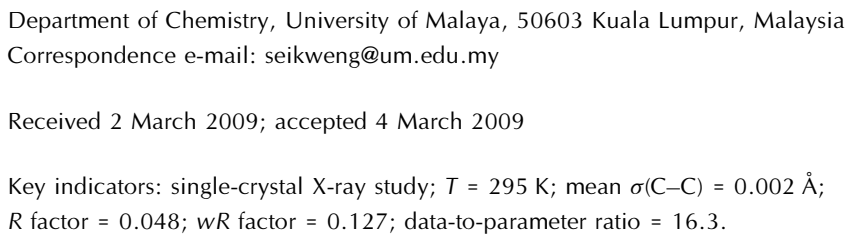

Department of Chemistry, University of Malaya, 50603 Kuala Lumpur, Malaysia Correspondence e-mail: seikweng@um.edu.my

Received 2 March 2009; accepted 4 March 2009

Key indicators: single-crystal X-ray study; $T=295 \mathrm{~K}$; mean $\sigma(\mathrm{C}-\mathrm{C})=0.002 \AA$; $R$ factor $=0.048 ; w R$ factor $=0.127 ;$ data-to-parameter ratio $=16.3$.

In the crystal structure of the title compound, $\mathrm{C}_{18} \mathrm{H}_{12} \mathrm{~N}_{2} \mathrm{O}$, the two fused rings are aligned at $64.2(1)^{\circ}$; the $\mathrm{C}-\mathrm{O}-\mathrm{C}$ angle is $118.73(12)^{\circ}$.

\section{Related literature}

For the crystal structure of 1-naphthyl quinoxalinyl ether, see: Hassan et al. (2009).<smiles>c1ccc2cc(Oc3cnc4ccccc4n3)ccc2c1</smiles>

\section{Experimental}

Crystal data
$\mathrm{C}_{18} \mathrm{H}_{12} \mathrm{~N}_{2} \mathrm{O}$
$M_{r}=272.30$

Monoclinic, $P 2_{1} / c$

$a=6.808$ (1) $\AA$

$b=7.609$ (1) $\AA$

$c=26.234(3) \AA$

$\beta=92.522(2)^{\circ}$

$V=1357.5(3) \AA^{3}$

$Z=4$

Mo $K \alpha$ radiation

$\mu=0.08 \mathrm{~mm}^{-1}$

$T=295 \mathrm{~K}$

$0.45 \times 0.15 \times 0.10 \mathrm{~mm}$

Data collection

Bruker SMART APEX

diffractometer

Absorption correction: none

7510 measured reflections

3094 independent reflections 1950 reflections with $I>2 \sigma(I)$ $R_{\text {int }}=0.032$

Refinement

$R\left[F^{2}>2 \sigma\left(F^{2}\right)\right]=0.048$

$w R\left(F^{2}\right)=0.127$

$S=1.04$

3094 reflections

190 parameters

$\mathrm{H}$-atom parameters constrained

$\Delta \rho_{\max }=0.17{\mathrm{e} \AA^{-3}}^{-3}$

$\Delta \rho_{\min }=-0.16{\mathrm{e} \AA^{-3}}^{-3}$

Data collection: APEX2 (Bruker, 2007); cell refinement: SAINT (Bruker, 2007); data reduction: $S A I N T$; program(s) used to solve structure: SHELXS97 (Sheldrick, 2008); program(s) used to refine structure: SHELXL97 (Sheldrick, 2008); molecular graphics: $X$ $S E E D$ (Barbour, 2001); software used to prepare material for publication: publCIF (Westrip, 2009).

We thank the University of Malaya for supporting this study (FS358/2008 A).

Supplementary data and figures for this paper are available from the IUCr electronic archives (Reference: TK2387).

\section{References}

Barbour, L. J. (2001). J. Supramol. Chem. 1, 189-191.

Bruker (2007). APEX2 and SAINT. Bruker AXS Inc., Madison, Wisconsin, USA.

Hassan, N. D., Tajuddin, H. A., Abdullah, Z. \& Ng, S. W. (2009). Acta Cryst. E65, o731.

Sheldrick, G. M. (2008). Acta Cryst. A64, 112-122.

Westrip, S. P. (2009). publCIF. In preparation. 


\section{supporting information}

Acta Cryst. (2009). E65, o732 [doi:10.1107/S1600536809007855]

\section{2-Naphthyl quinoxalin-2-yl ether}

\section{Noor Doha Hassan, Hairul Anuar Tajuddin, Zanariah Abdullah and Seik Weng Ng}

\section{S1. Experimental}

2-Naphthol (2.88 g, $20 \mathrm{mmol})$ was mixed with sodium hydroxide $(0.08 \mathrm{~g}, 20 \mathrm{mmol})$ in several drops of water. The water was then evaporated. The paste was heated with 2-chloroquinoxaline $(3.29 \mathrm{~g}, 20 \mathrm{mmol})$ at $423-433 \mathrm{~K}$ for $6 \mathrm{~h}$. The product was dissolved in water and the solution extracted with chloroform. The chloroform phase was dried over sodium sulfate; the evaporation of the solvent gave a product that was recrystallized from an ethyl acetate/hexane.

\section{S2. Refinement}

Carbon-bound H-atoms were placed in calculated positions ( $\mathrm{C}-\mathrm{H} 0.93 \AA)$ and were included in the refinement in the riding model approximation, with $U(\mathrm{H})$ set to $1.2 U_{\mathrm{eq}}(\mathrm{C})$.

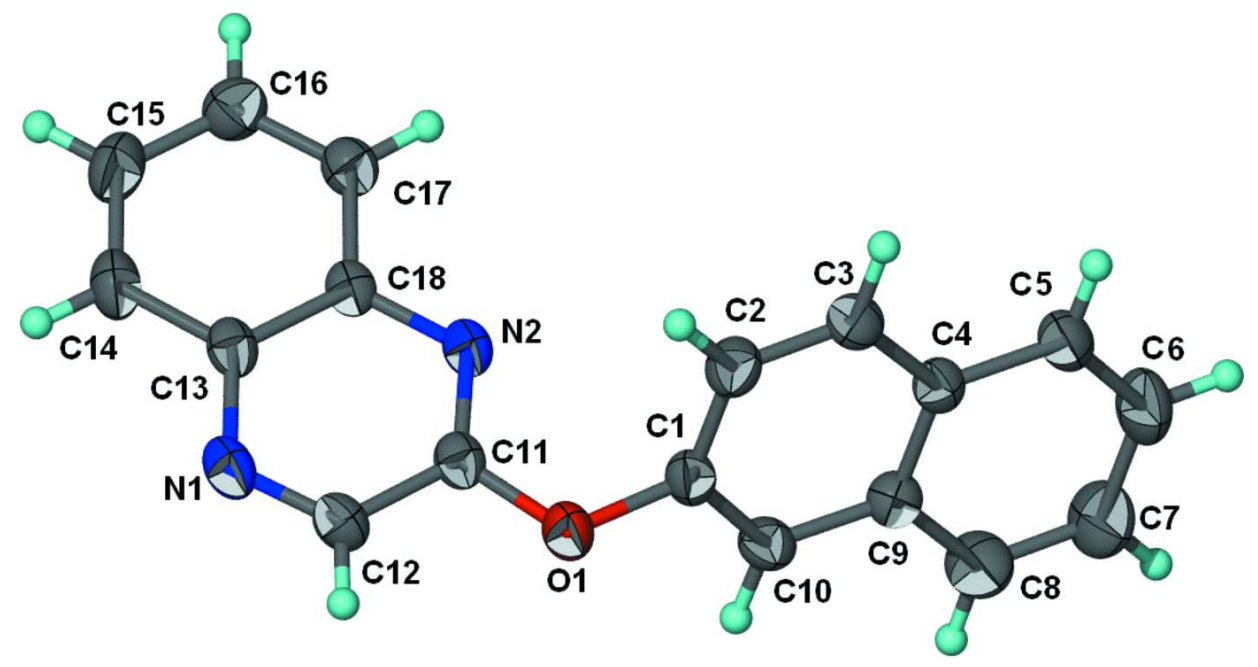

\section{Figure 1}

Thermal ellipsoid plot (Barbour, 2001) of the molecule of $\mathrm{C}_{18} \mathrm{H}_{12} \mathrm{~N}_{2} \mathrm{O}$ at the $50 \%$ probability level. Hydrogen atoms are drawn as spheres of arbitrary radius.

\section{2-Naphthyl quinoxalin-2-yl ether}

Crystal data

$\mathrm{C}_{18} \mathrm{H}_{12} \mathrm{~N}_{2} \mathrm{O}$

$M_{r}=272.30$

Monoclinic, $P 2_{1} / c$

Hall symbol: -P $2 \mathrm{ybc}$

$a=6.808$ (1) $\AA$

$b=7.609$ (1) $\AA$

$c=26.234(3) \AA$

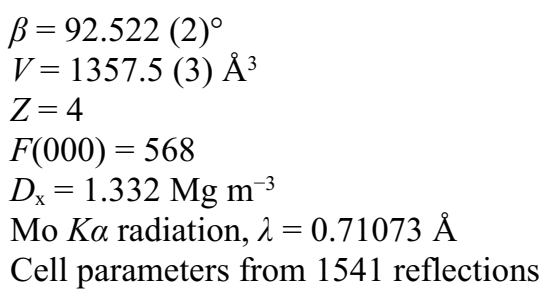


$\theta=2.7-28.2^{\circ}$

$\mu=0.08 \mathrm{~mm}^{-1}$

$T=295 \mathrm{~K}$

\section{Data collection}

\section{Bruker SMART APEX}

diffractometer

Radiation source: fine-focus sealed tube

Graphite monochromator

$\omega$ scans

7510 measured reflections

3094 independent reflections

\section{Refinement}

Refinement on $F^{2}$

Least-squares matrix: full

$R\left[F^{2}>2 \sigma\left(F^{2}\right)\right]=0.048$

$w R\left(F^{2}\right)=0.127$

$S=1.04$

3094 reflections

190 parameters

0 restraints

Primary atom site location: structure-invariant direct methods
Block, colorless

$0.45 \times 0.15 \times 0.10 \mathrm{~mm}$

1950 reflections with $I>2 \sigma(I)$

$R_{\text {int }}=0.032$

$\theta_{\max }=27.5^{\circ}, \theta_{\min }=2.8^{\circ}$

$h=-6 \rightarrow 8$

$k=-9 \rightarrow 8$

$l=-28 \rightarrow 34$

Secondary atom site location: difference Fourier map

Hydrogen site location: inferred from neighbouring sites

$\mathrm{H}$-atom parameters constrained

$w=1 /\left[\sigma^{2}\left(F_{\mathrm{o}}{ }^{2}\right)+(0.0544 P)^{2}+0.1253 P\right]$

where $P=\left(F_{\mathrm{o}}^{2}+2 F_{\mathrm{c}}^{2}\right) / 3$

$(\Delta / \sigma)_{\max }=0.001$

$\Delta \rho_{\max }=0.17$ e $\AA^{-3}$

$\Delta \rho_{\min }=-0.16$ e $\AA^{-3}$

Fractional atomic coordinates and isotropic or equivalent isotropic displacement parameters $\left(\AA^{2}\right)$

\begin{tabular}{lllll}
\hline & $x$ & $y$ & $z$ & $U_{\text {iso }} * / U_{\text {eq }}$ \\
\hline O1 & $0.11303(15)$ & $0.36756(17)$ & $0.59259(4)$ & $0.0477(3)$ \\
$\mathrm{N} 1$ & $-0.13352(19)$ & $0.2389(2)$ & $0.47654(5)$ & $0.0459(4)$ \\
$\mathrm{N} 2$ & $0.25074(18)$ & $0.24104(18)$ & $0.52218(5)$ & $0.0379(3)$ \\
$\mathrm{C} 1$ & $0.2995(2)$ & $0.3759(2)$ & $0.61786(6)$ & $0.0392(4)$ \\
$\mathrm{C} 2$ & $0.4441(2)$ & $0.4866(2)$ & $0.59948(6)$ & $0.0447(4)$ \\
$\mathrm{H} 2$ & 0.4210 & 0.5499 & 0.5695 & $0.054^{*}$ \\
$\mathrm{C} 3$ & $0.6192(2)$ & $0.5003(2)$ & $0.62616(6)$ & $0.0435(4)$ \\
$\mathrm{H} 3$ & 0.7168 & 0.5723 & 0.6138 & $0.052^{*}$ \\
C4 & $0.6559(2)$ & $0.4077(2)$ & $0.67216(6)$ & $0.0371(4)$ \\
C5 & $0.5053(2)$ & $0.3003(2)$ & $0.69082(6)$ & $0.0394(4)$ \\
C6 & $0.3253(2)$ & $0.2858(2)$ & $0.66207(6)$ & $0.0412(4)$ \\
H6 & 0.2253 & 0.2147 & 0.6735 & $0.049 *$ \\
C7 & $0.8364(2)$ & $0.4201(3)$ & $0.70077(6)$ & $0.0483(5)$ \\
H7 & 0.9372 & 0.4892 & 0.6888 & $0.058^{*}$ \\
C8 & $0.8642(3)$ & $0.3322(3)$ & $0.74553(7)$ & $0.0618(6)$ \\
H8 & 0.9839 & 0.3414 & 0.7638 & $0.074 *$ \\
C9 & $0.7154(3)$ & $0.2285(3)$ & $0.76428(7)$ & $0.0694(6)$ \\
H9 & 0.7355 & 0.1704 & 0.7953 & $0.083^{*}$ \\
C10 & $0.5402(3)$ & $0.2116(3)$ & $0.73751(7)$ & $0.0593(5)$ \\
H10 & 0.4424 & 0.1406 & 0.7502 & $0.071^{*}$ \\
C11 & $0.0985(2)$ & $0.3003(2)$ & $0.54445(6)$ & $0.0375(4)$ \\
C12 & $-0.0954(2)$ & $0.2999(2)$ & $0.52209(6)$ & $0.0439(4)$ \\
H12 & -0.1976 & 0.3445 & 0.5406 & $0.053^{*}$ \\
& & & &
\end{tabular}


supporting information

\begin{tabular}{lllll} 
C13 & $0.0233(2)$ & $0.1738(2)$ & $0.45104(6)$ & $0.0389(4)$ \\
C14 & $-0.0076(3)$ & $0.1015(3)$ & $0.40208(6)$ & $0.0520(5)$ \\
H14 & -0.1333 & 0.1008 & 0.3867 & $0.062^{*}$ \\
C15 & $0.1453(3)$ & $0.0325(3)$ & $0.37707(7)$ & $0.0561(5)$ \\
H15 & 0.1237 & -0.0157 & 0.3447 & $0.067^{*}$ \\
C16 & $0.3351(3)$ & $0.0340(2)$ & $0.39983(7)$ & $0.0524(5)$ \\
H16 & 0.4385 & -0.0139 & 0.3824 & $0.063^{*}$ \\
C17 & $0.3705(2)$ & $0.1044(2)$ & $0.44705(6)$ & $0.0430(4)$ \\
H17 & 0.4977 & 0.1063 & 0.4615 & $0.052^{*}$ \\
C18 & $0.2149(2)$ & $0.1739(2)$ & $0.47388(5)$ & $0.0348(4)$ \\
\hline
\end{tabular}

Atomic displacement parameters $\left(\AA^{2}\right)$

\begin{tabular}{lllllll}
\hline & $U^{11}$ & $U^{22}$ & $U^{33}$ & $U^{12}$ & $U^{13}$ & $U^{23}$ \\
\hline $\mathrm{O} 1$ & $0.0342(6)$ & $0.0636(9)$ & $0.0449(6)$ & $0.0032(5)$ & $-0.0035(5)$ & $-0.0096(6)$ \\
$\mathrm{N} 1$ & $0.0344(8)$ & $0.0508(10)$ & $0.0517(8)$ & $-0.0015(6)$ & $-0.0080(6)$ & $0.0037(7)$ \\
$\mathrm{N} 2$ & $0.0336(7)$ & $0.0387(8)$ & $0.0409(7)$ & $-0.0003(6)$ & $-0.0048(5)$ & $0.0016(6)$ \\
$\mathrm{C} 1$ & $0.0348(9)$ & $0.0424(11)$ & $0.0402(9)$ & $0.0010(7)$ & $-0.0013(7)$ & $-0.0076(7)$ \\
$\mathrm{C} 2$ & $0.0497(10)$ & $0.0453(11)$ & $0.0389(9)$ & $-0.0025(8)$ & $-0.0017(7)$ & $0.0046(8)$ \\
$\mathrm{C} 3$ & $0.0441(9)$ & $0.0424(11)$ & $0.0441(9)$ & $-0.0095(8)$ & $0.0033(7)$ & $0.0009(8)$ \\
$\mathrm{C} 4$ & $0.0376(8)$ & $0.0339(10)$ & $0.0398(8)$ & $0.0010(7)$ & $0.0004(6)$ & $-0.0055(7)$ \\
$\mathrm{C} 5$ & $0.0420(9)$ & $0.0356(10)$ & $0.0404(9)$ & $0.0010(7)$ & $0.0010(7)$ & $0.0008(7)$ \\
$\mathrm{C} 6$ & $0.0392(9)$ & $0.0390(11)$ & $0.0457(9)$ & $-0.0056(7)$ & $0.0052(7)$ & $-0.0005(8)$ \\
$\mathrm{C} 7$ & $0.0382(9)$ & $0.0534(13)$ & $0.0529(10)$ & $-0.0002(8)$ & $-0.0015(7)$ & $-0.0099(9)$ \\
$\mathrm{C} 8$ & $0.0510(12)$ & $0.0723(16)$ & $0.0603(12)$ & $0.0077(10)$ & $-0.0177(9)$ & $-0.0017(10)$ \\
$\mathrm{C} 9$ & $0.0681(15)$ & $0.0816(17)$ & $0.0571(12)$ & $0.0052(12)$ & $-0.0130(10)$ & $0.0210(11)$ \\
C10 & $0.0594(12)$ & $0.0617(15)$ & $0.0566(11)$ & $-0.0039(10)$ & $-0.0004(9)$ & $0.0194(10)$ \\
C11 & $0.0366(9)$ & $0.0351(10)$ & $0.0404(8)$ & $-0.0013(7)$ & $-0.0033(7)$ & $0.0026(7)$ \\
C12 & $0.0332(8)$ & $0.0476(11)$ & $0.0506(10)$ & $0.0030(8)$ & $-0.0019(7)$ & $0.0007(8)$ \\
C13 & $0.0387(9)$ & $0.0341(10)$ & $0.0431(9)$ & $-0.0050(7)$ & $-0.0058(7)$ & $0.0045(7)$ \\
C14 & $0.0502(11)$ & $0.0557(13)$ & $0.0486(10)$ & $-0.0079(9)$ & $-0.0138(8)$ & $-0.0009(9)$ \\
C15 & $0.0689(13)$ & $0.0558(14)$ & $0.0431(10)$ & $-0.0075(10)$ & $-0.0046(9)$ & $-0.0087(9)$ \\
C16 & $0.0559(11)$ & $0.0509(13)$ & $0.0507(10)$ & $0.0038(9)$ & $0.0047(8)$ & $-0.0040(9)$ \\
C17 & $0.0401(9)$ & $0.0415(11)$ & $0.0473(9)$ & $0.0018(7)$ & $-0.0013(7)$ & $0.0025(8)$ \\
C18 & $0.0360(8)$ & $0.0295(9)$ & $0.0386(8)$ & $-0.0029(7)$ & $-0.0036(6)$ & $0.0060(7)$ \\
& & & & & & \\
\hline
\end{tabular}

Geometric parameters $\left(\stackrel{A}{\circ}{ }^{\circ}\right)$

\begin{tabular}{llll}
\hline $\mathrm{O} 1-\mathrm{C} 11$ & $1.3622(18)$ & $\mathrm{C} 7-\mathrm{H} 7$ & 0.9300 \\
$\mathrm{O} 1-\mathrm{C} 1$ & $1.4073(18)$ & $\mathrm{C} 8-\mathrm{C} 9$ & $1.391(3)$ \\
$\mathrm{N} 1-\mathrm{C} 12$ & $1.297(2)$ & $\mathrm{C} 8-\mathrm{H} 8$ & 0.9300 \\
$\mathrm{~N} 1-\mathrm{C} 13$ & $1.377(2)$ & $\mathrm{C} 9-\mathrm{C} 10$ & $1.363(3)$ \\
$\mathrm{N} 2-\mathrm{C} 11$ & $1.2928(19)$ & $\mathrm{C} 9-\mathrm{H} 9$ & 0.9300 \\
$\mathrm{~N} 2-\mathrm{C} 18$ & $1.3778(19)$ & $\mathrm{C} 10-\mathrm{H} 10$ & 0.9300 \\
$\mathrm{C} 1-\mathrm{C} 6$ & $1.352(2)$ & $\mathrm{C} 11-\mathrm{C} 12$ & $1.421(2)$ \\
$\mathrm{C} 1-\mathrm{C} 2$ & $1.398(2)$ & $\mathrm{C} 12-\mathrm{H} 12$ & 0.9300 \\
$\mathrm{C} 2-\mathrm{C} 3$ & $1.359(2)$ & $\mathrm{C} 13-\mathrm{C} 14$ & $1.405(2)$ \\
$\mathrm{C} 2-\mathrm{H} 2$ & 0.9300 & $\mathrm{C} 13-\mathrm{C} 18$ & $1.411(2)$
\end{tabular}




\begin{tabular}{|c|c|c|c|}
\hline $\mathrm{C} 3-\mathrm{C} 4$ & $1.410(2)$ & $\mathrm{C} 14-\mathrm{C} 15$ & $1.360(3)$ \\
\hline $\mathrm{C} 3-\mathrm{H} 3$ & 0.9300 & $\mathrm{C} 14-\mathrm{H} 14$ & 0.9300 \\
\hline $\mathrm{C} 4-\mathrm{C} 5$ & $1.415(2)$ & $\mathrm{C} 15-\mathrm{C} 16$ & $1.399(2)$ \\
\hline $\mathrm{C} 4-\mathrm{C} 7$ & $1.415(2)$ & $\mathrm{C} 15-\mathrm{H} 15$ & 0.9300 \\
\hline $\mathrm{C} 5-\mathrm{C} 10$ & $1.410(2)$ & $\mathrm{C} 16-\mathrm{C} 17$ & $1.361(2)$ \\
\hline $\mathrm{C} 5-\mathrm{C} 6$ & $1.414(2)$ & $\mathrm{C} 16-\mathrm{H} 16$ & 0.9300 \\
\hline $\mathrm{C} 6-\mathrm{H} 6$ & 0.9300 & $\mathrm{C} 17-\mathrm{C} 18$ & $1.401(2)$ \\
\hline $\mathrm{C} 7-\mathrm{C} 8$ & $1.357(2)$ & $\mathrm{C} 17-\mathrm{H} 17$ & 0.9300 \\
\hline $\mathrm{C} 11-\mathrm{O} 1-\mathrm{C} 1$ & $118.73(12)$ & $\mathrm{C} 8-\mathrm{C} 9-\mathrm{H} 9$ & 119.8 \\
\hline $\mathrm{C} 12-\mathrm{N} 1-\mathrm{C} 13$ & $116.62(13)$ & $\mathrm{C} 9-\mathrm{C} 10-\mathrm{C} 5$ & $120.83(19)$ \\
\hline $\mathrm{C} 11-\mathrm{N} 2-\mathrm{C} 18$ & $115.58(13)$ & $\mathrm{C} 9-\mathrm{C} 10-\mathrm{H} 10$ & 119.6 \\
\hline $\mathrm{C} 6-\mathrm{C} 1-\mathrm{C} 2$ & $122.30(15)$ & $\mathrm{C} 5-\mathrm{C} 10-\mathrm{H} 10$ & 119.6 \\
\hline $\mathrm{C} 6-\mathrm{C} 1-\mathrm{O} 1$ & $117.50(15)$ & $\mathrm{N} 2-\mathrm{C} 11-\mathrm{O} 1$ & $121.55(13)$ \\
\hline $\mathrm{C} 2-\mathrm{C} 1-\mathrm{O} 1$ & $119.95(14)$ & $\mathrm{N} 2-\mathrm{C} 11-\mathrm{C} 12$ & $124.14(15)$ \\
\hline $\mathrm{C} 3-\mathrm{C} 2-\mathrm{C} 1$ & $118.92(15)$ & $\mathrm{O} 1-\mathrm{C} 11-\mathrm{C} 12$ & $114.31(14)$ \\
\hline $\mathrm{C} 3-\mathrm{C} 2-\mathrm{H} 2$ & 120.5 & $\mathrm{~N} 1-\mathrm{C} 12-\mathrm{C} 11$ & $121.72(15)$ \\
\hline $\mathrm{C} 1-\mathrm{C} 2-\mathrm{H} 2$ & 120.5 & $\mathrm{~N} 1-\mathrm{C} 12-\mathrm{H} 12$ & 119.1 \\
\hline $\mathrm{C} 2-\mathrm{C} 3-\mathrm{C} 4$ & $121.39(16)$ & $\mathrm{C} 11-\mathrm{C} 12-\mathrm{H} 12$ & 119.1 \\
\hline $\mathrm{C} 2-\mathrm{C} 3-\mathrm{H} 3$ & 119.3 & $\mathrm{~N} 1-\mathrm{C} 13-\mathrm{C} 14$ & $119.82(15)$ \\
\hline $\mathrm{C} 4-\mathrm{C} 3-\mathrm{H} 3$ & 119.3 & $\mathrm{~N} 1-\mathrm{C} 13-\mathrm{C} 18$ & $121.02(14)$ \\
\hline $\mathrm{C} 3-\mathrm{C} 4-\mathrm{C} 5$ & $118.72(14)$ & $\mathrm{C} 14-\mathrm{C} 13-\mathrm{C} 18$ & $119.14(15)$ \\
\hline $\mathrm{C} 3-\mathrm{C} 4-\mathrm{C} 7$ & $122.55(16)$ & $\mathrm{C} 15-\mathrm{C} 14-\mathrm{C} 13$ & $120.36(16)$ \\
\hline $\mathrm{C} 5-\mathrm{C} 4-\mathrm{C} 7$ & $118.72(15)$ & $\mathrm{C} 15-\mathrm{C} 14-\mathrm{H} 14$ & 119.8 \\
\hline $\mathrm{C} 10-\mathrm{C} 5-\mathrm{C} 4$ & $118.70(15)$ & $\mathrm{C} 13-\mathrm{C} 14-\mathrm{H} 14$ & 119.8 \\
\hline $\mathrm{C} 10-\mathrm{C} 5-\mathrm{C} 6$ & $122.33(16)$ & $\mathrm{C} 14-\mathrm{C} 15-\mathrm{C} 16$ & $120.19(16)$ \\
\hline $\mathrm{C} 4-\mathrm{C} 5-\mathrm{C} 6$ & $118.97(14)$ & $\mathrm{C} 14-\mathrm{C} 15-\mathrm{H} 15$ & 119.9 \\
\hline $\mathrm{C} 1-\mathrm{C} 6-\mathrm{C} 5$ & $119.65(15)$ & $\mathrm{C} 16-\mathrm{C} 15-\mathrm{H} 15$ & 119.9 \\
\hline $\mathrm{C} 1-\mathrm{C} 6-\mathrm{H} 6$ & 120.2 & $\mathrm{C} 17-\mathrm{C} 16-\mathrm{C} 15$ & $120.98(17)$ \\
\hline $\mathrm{C} 5-\mathrm{C} 6-\mathrm{H} 6$ & 120.2 & $\mathrm{C} 17-\mathrm{C} 16-\mathrm{H} 16$ & 119.5 \\
\hline $\mathrm{C} 8-\mathrm{C} 7-\mathrm{C} 4$ & $120.72(17)$ & $\mathrm{C} 15-\mathrm{C} 16-\mathrm{H} 16$ & 119.5 \\
\hline $\mathrm{C} 8-\mathrm{C} 7-\mathrm{H} 7$ & 119.6 & $\mathrm{C} 16-\mathrm{C} 17-\mathrm{C} 18$ & $119.89(16)$ \\
\hline $\mathrm{C} 4-\mathrm{C} 7-\mathrm{H} 7$ & 119.6 & $\mathrm{C} 16-\mathrm{C} 17-\mathrm{H} 17$ & 120.1 \\
\hline $\mathrm{C} 7-\mathrm{C} 8-\mathrm{C} 9$ & $120.65(17)$ & $\mathrm{C} 18-\mathrm{C} 17-\mathrm{H} 17$ & 120.1 \\
\hline $\mathrm{C} 7-\mathrm{C} 8-\mathrm{H} 8$ & 119.7 & $\mathrm{~N} 2-\mathrm{C} 18-\mathrm{C} 17$ & $119.67(14)$ \\
\hline $\mathrm{C} 9-\mathrm{C} 8-\mathrm{H} 8$ & 119.7 & $\mathrm{~N} 2-\mathrm{C} 18-\mathrm{C} 13$ & $120.91(14)$ \\
\hline $\mathrm{C} 10-\mathrm{C} 9-\mathrm{C} 8$ & $120.38(18)$ & $\mathrm{C} 17-\mathrm{C} 18-\mathrm{C} 13$ & $119.43(14)$ \\
\hline $\mathrm{C} 10-\mathrm{C} 9-\mathrm{H} 9$ & 119.8 & & \\
\hline $\mathrm{C} 11-\mathrm{O} 1-\mathrm{C} 1-\mathrm{C} 6$ & $-119.60(16)$ & $\mathrm{C} 18-\mathrm{N} 2-\mathrm{C} 11-\mathrm{O} 1$ & $178.93(14)$ \\
\hline $\mathrm{C} 11-\mathrm{O} 1-\mathrm{C} 1-\mathrm{C} 2$ & $65.9(2)$ & $\mathrm{C} 18-\mathrm{N} 2-\mathrm{C} 11-\mathrm{C} 12$ & $-0.3(2)$ \\
\hline $\mathrm{C} 6-\mathrm{C} 1-\mathrm{C} 2-\mathrm{C} 3$ & $2.0(3)$ & $\mathrm{C} 1-\mathrm{O} 1-\mathrm{C} 11-\mathrm{N} 2$ & $1.5(2)$ \\
\hline $\mathrm{O} 1-\mathrm{C} 1-\mathrm{C} 2-\mathrm{C} 3$ & $176.20(15)$ & $\mathrm{C} 1-\mathrm{O} 1-\mathrm{C} 11-\mathrm{C} 12$ & $-179.20(15)$ \\
\hline $\mathrm{C} 1-\mathrm{C} 2-\mathrm{C} 3-\mathrm{C} 4$ & $-1.1(3)$ & $\mathrm{C} 13-\mathrm{N} 1-\mathrm{C} 12-\mathrm{C} 11$ & $0.3(2)$ \\
\hline $\mathrm{C} 2-\mathrm{C} 3-\mathrm{C} 4-\mathrm{C} 5$ & $-0.7(2)$ & $\mathrm{N} 2-\mathrm{C} 11-\mathrm{C} 12-\mathrm{N} 1$ & $-0.2(3)$ \\
\hline $\mathrm{C} 2-\mathrm{C} 3-\mathrm{C} 4-\mathrm{C} 7$ & $-179.77(16)$ & $\mathrm{O} 1-\mathrm{C} 11-\mathrm{C} 12-\mathrm{N} 1$ & $-179.53(15)$ \\
\hline $\mathrm{C} 3-\mathrm{C} 4-\mathrm{C} 5-\mathrm{C} 10$ & $-178.29(16)$ & $\mathrm{C} 12-\mathrm{N} 1-\mathrm{C} 13-\mathrm{C} 14$ & $178.37(16)$ \\
\hline $\mathrm{C} 7-\mathrm{C} 4-\mathrm{C} 5-\mathrm{C} 10$ & $0.8(2)$ & $\mathrm{C} 12-\mathrm{N} 1-\mathrm{C} 13-\mathrm{C} 18$ & $0.1(2)$ \\
\hline
\end{tabular}




$\begin{array}{llll}\mathrm{C} 3-\mathrm{C} 4-\mathrm{C} 5-\mathrm{C} 6 & 1.7(2) & \mathrm{N} 1-\mathrm{C} 13-\mathrm{C} 14-\mathrm{C} 15 & -178.25(17) \\ \mathrm{C} 7-\mathrm{C} 4-\mathrm{C} 5-\mathrm{C} 6 & -179.25(15) & \mathrm{C} 18-\mathrm{C} 13-\mathrm{C} 14-\mathrm{C} 15 & 0.0(3) \\ \mathrm{C} 2-\mathrm{C} 1-\mathrm{C} 6-\mathrm{C} 5 & -1.1(3) & \mathrm{C} 13-\mathrm{C} 14-\mathrm{C} 15-\mathrm{C} 16 & -0.4(3) \\ \mathrm{O} 1-\mathrm{C} 1-\mathrm{C} 6-\mathrm{C} 5 & -175.37(14) & \mathrm{C} 14-\mathrm{C} 15-\mathrm{C} 16-\mathrm{C} 17 & -0.2(3) \\ \mathrm{C} 10-\mathrm{C} 5-\mathrm{C} 6-\mathrm{C} 1 & 179.15(16) & \mathrm{C} 15-\mathrm{C} 16-\mathrm{C} 17-\mathrm{C} 18 & 1.1(3) \\ \mathrm{C} 4-\mathrm{C} 5-\mathrm{C} 6-\mathrm{C} 1 & -0.8(2) & \mathrm{C} 11-\mathrm{N} 2-\mathrm{C} 18-\mathrm{C} 17 & -179.07(14) \\ \mathrm{C} 3-\mathrm{C} 4-\mathrm{C} 7-\mathrm{C} 8 & 178.35(17) & \mathrm{C} 11-\mathrm{N} 2-\mathrm{C} 18-\mathrm{C} 13 & 0.8(2) \\ \mathrm{C} 5-\mathrm{C} 4-\mathrm{C} 7-\mathrm{C} 8 & -0.7(3) & \mathrm{C} 16-\mathrm{C} 17-\mathrm{C} 18-\mathrm{N} 2 & 178.38(15) \\ \mathrm{C} 4-\mathrm{C} 7-\mathrm{C} 8-\mathrm{C} 9 & -0.2(3) & \mathrm{C} 16-\mathrm{C} 17-\mathrm{C} 18-\mathrm{C} 13 & -1.5(2) \\ \mathrm{C} 7-\mathrm{C} 8-\mathrm{C} 9-\mathrm{C} 10 & 1.0(3) & \mathrm{N} 1-\mathrm{C} 13-\mathrm{C} 18-\mathrm{N} 2 & -0.7(2) \\ \mathrm{C} 8-\mathrm{C} 9-\mathrm{C} 10-\mathrm{C} 5 & -0.9(3) & \mathrm{C} 14-\mathrm{C} 13-\mathrm{C} 18-\mathrm{N} 2 & -178.94(15) \\ \mathrm{C} 4-\mathrm{C} 5-\mathrm{C} 10-\mathrm{C} 9 & 0.0(3) & \mathrm{N} 1-\mathrm{C} 13-\mathrm{C} 18-\mathrm{C} 17 & 179.13(15) \\ \mathrm{C} 6-\mathrm{C} 5-\mathrm{C} 10-\mathrm{C} 9 & -179.97(18) & \mathrm{C} 14-\mathrm{C} 13-\mathrm{C} 18-\mathrm{C} 17 & 0.9(2)\end{array}$

